\title{
Effects of context-aware patient guidance on blood pressure self- measurement adherence levels
}

Health Informatics Journal 2019, Vol. 25(2) 417-428

(C) The Author(s) 2017

Article reuse guidelines: sagepub.com/journals-permissions DOI: $10.1177 / 14604582177 / 7073$ journals.sagepub.com/home/jhi

\section{Camilla Lindahl}

Aarhus University Hospital, Denmark

\section{Stefan Wagner}

Aarhus University, Denmark

\section{Niels Uldbjerg and Jacob Mørup Schlütter}

Aarhus University Hospital, Denmark

\section{Olav Bertelsen}

Aarhus University, Denmark

\section{Puk Sandager}

Aarhus University Hospital, Denmark

\begin{abstract}
Hypertensive disease and preeclampsia are serious medical disorders affecting pregnancy. Screening in early pregnancy may identify women at risk of developing hypertensive disease and enable prophylactic treatment. Accurate blood pressure measurement is an important part of this screening. The aim of this study was to investigate whether patients, with the aid of a context-aware sensor-based blood pressure self-measurement system, were able to correctly self-measure their blood pressure adhering to internationally established recommendations. Furthermore, to evaluate patient acceptance levels of performing self-measurements using context-aware adherence aids for guidance. A total of 100 pregnant women attending a routine ultrasound, at gestational week 12, at the outpatient clinic of the Department of Obstetrics and Gynecology, Aarhus University Hospital, were recruited in the waiting room before the scheduled scan. Blood pressure self-measurement was performed using a blood pressure selfmeasurement system called ValidAid consisting of a clinically approved blood pressure device, a sensor chair registering rest-time, back-supported, legs-crossed, and ambient noise levels respectively recording participant compliance, as well as a touch screen-based computer application with an interactive user
\end{abstract}

Corresponding author:

Stefan Wagner, Department of Engineering, Aarhus University, Finlandsgade 22, 8200 Aarhus N, Denmark.

Email: sw@eng.au.dk 
interface for patient guidance and feedback, as well as a built-in decision support system. Acceptance of the automated self-measurement was evaluated by a questionnaire. In all, 99 percent followed the instructions with regard to both rest time and not talking. For both of these, ValidAid offered interactive and context-aware guidance. The recommendation of keeping legs uncrossed was only adhered to in 69 percent of measurements and back supported in 35 percent of measurements. For both of these, no interactive guidance was provided. The majority of the participants, 93 percent, felt comfortable using self-measurement equipment, while a minority of 8 percent would have preferred personnel-assisted measurements. The majority of participants were able to take reliable blood pressure self-measurements. Results indicate that recommendations that were not actively enforced were not followed to the same extent as those that were enforced. Thus, providing interactive context-aware guidance for all recommendations should be considered in the future. Furthermore, we found patient acceptance levels of performing self-measurements to be overall positive.

\section{Keywords}

clinical decision-making, context-aware sensors, decision-support systems, ehealth, hypertension, IT healthcare evaluation, pervasive technologies, preeclampsia

\section{Introduction}

Sensor-based systems could be useful for supporting more reliable and efficient self-measurement of health parameters in the outpatient clinical setting in the future. However, this area has not yet been studied for clinical effect, including feasibility and acceptability of the patients for being part of such a system. This article investigates the feasibility and acceptability of an intelligent selfmeasurement station based on a combination of context-aware and medical sensors providing interactive patient guidance and registering additional context information that is important for pregnant women that needs to self-measure their blood pressure (BP) as part of a screening process for preeclampsia (PE).

Hypertensive disease and PE are some of the most common medical disorders affecting pregnancy today. ${ }^{1,2}$ Approximately $2-8$ percent of all pregnancies are complicated by $\mathrm{PE},{ }^{3}$ a condition which is associated with an increased risk of fetal growth restriction, fetal death, and maternal morbidity including generalized seizures, intracerebral hemorrhage, and severe/lethal effects of multiple organ systems. ${ }^{1}$

PE is defined as elevated BP levels of over 140/90 $\mathrm{mm} \mathrm{Hg}$ after gestational week 20, accompanied by proteinuria (an excess of serum proteins in urine) ${ }^{4}$ Early diagnosis is essential to reduce the risk of complications. ${ }^{5}$

Recent studies indicate that screening of asymptomatic pregnant women in their first trimester of pregnancy, and prophylactic medical treatment, can reduce the risk of developing severe PE. ${ }^{5-11}$

Accurate BP measurement is an important part of this screening and in order to be valid for diagnostic use, a range of recommendations must be followed. ${ }^{12,13}$ Recommendations include: a quiet setting before and during measurements, being rested for $5 \mathrm{~min}$ before first measurement, not moving or talking, being correctly seated, legs not crossed, back supported, feet flat on the floor, arm supported at heart level, and finally correct cuff size and mounting. Screening of pregnant women in their first trimester will generally require increased resources of trained personnel to perform BP measurements.

Existing BP devices do not track behavioral data in order to measure adherence/compliance to recommendations. It is necessary for direct observation and intervention by staff in order to manage the quality of measurements, as there are no other means to validate measurements. Blood 
pressure self-measurement (BPSM) could save on staff resources and may perhaps also help reduce the risk of white coat hypertension, which is a well-known source of bias in 20 percent of patients visiting a clinic. ${ }^{13,14}$

White coat hypertension is a psychological anxiety-induced phenomena, which generally results in artificially high BP, compared with the actual BP measurements of the patients. ${ }^{13}$ There have, in recent years, been studies evaluating the ability of patients to self-measure BP and this has proved to be challenging. ${ }^{15}$

The aim of this study was to investigate whether patients, with the aid of a context-aware BPSM system, are able to correctly self-measure BP adhering to internationally established BP measurement recommendations and to evaluate patient acceptance levels of performing self-measurements using context-aware adherence aids for guidance.

\section{Materials and methods}

\section{Study design}

We included 100 pregnant women attending routine ultrasound, at gestational week 12, at the Department of Obstetrics and Gynecology, Aarhus University Hospital. Participants were approached in the waiting room, before the scheduled scan. Verbal and written information regarding the study purpose and structure was given to all participants. Women who had a normal first trimester scan, and who accepted to participate, were included in the study. Twin pregnancies were excluded. BP measurements were performed using a BP station, ValidAid. The ValidAid system was also used to gather information on BP measurements and relevant context parameters.

Patient age and actual gestational week were also recorded. Upon completion of the three measurements, each patient received a questionnaire to evaluate the acceptance of self-measurement. The questionnaire included seven questions, where participants were asked to score the experience of using the ValidAid system. Scores were divided into six categories; strongly agree, partly agree, neither-or, partly disagree, strongly disagree, and do not know.

\section{ValidAid system}

The ValidAid system (Figure 1) was developed by The Department of Engineering, at Aarhus University, as a research system for exploring patient adherence during self-measurement of BP. The ValidAid platform is an automatic context-aware system for capturing self-measured BP data, combined with data regarding the patient's adherence to the instructions according to the international recommendations regarding BP measurement. ${ }^{16}$ Two of the recommendations were actively enforced, rest time and not talking, while keeping legs uncrossed and back supported were not actively enforced.

The ValidAid system runs on a tablet computer (ASUS Eee EP121, Asus Inc., Taiwan) that integrates the BP device and sensor chair components, provides a user interface for the users, as well as performs audio classification of the data obtained. The ValidAid system uses a clinically approved BP device (A\&D Digital BP Monitor UA-767PBT, A\&D Company Limited, Japan), a sensor chair with built-in piezo-resistive sensors (FlexiForce A201 piezo-resistive sensor, TekScan Inc., USA) for registering leg placement and correct seating, including whether the back is supported.

The user interface consists of a screen guiding the patient through a series of three measurements (Figure 2). This is done through a countdown timer providing an indication of whether 


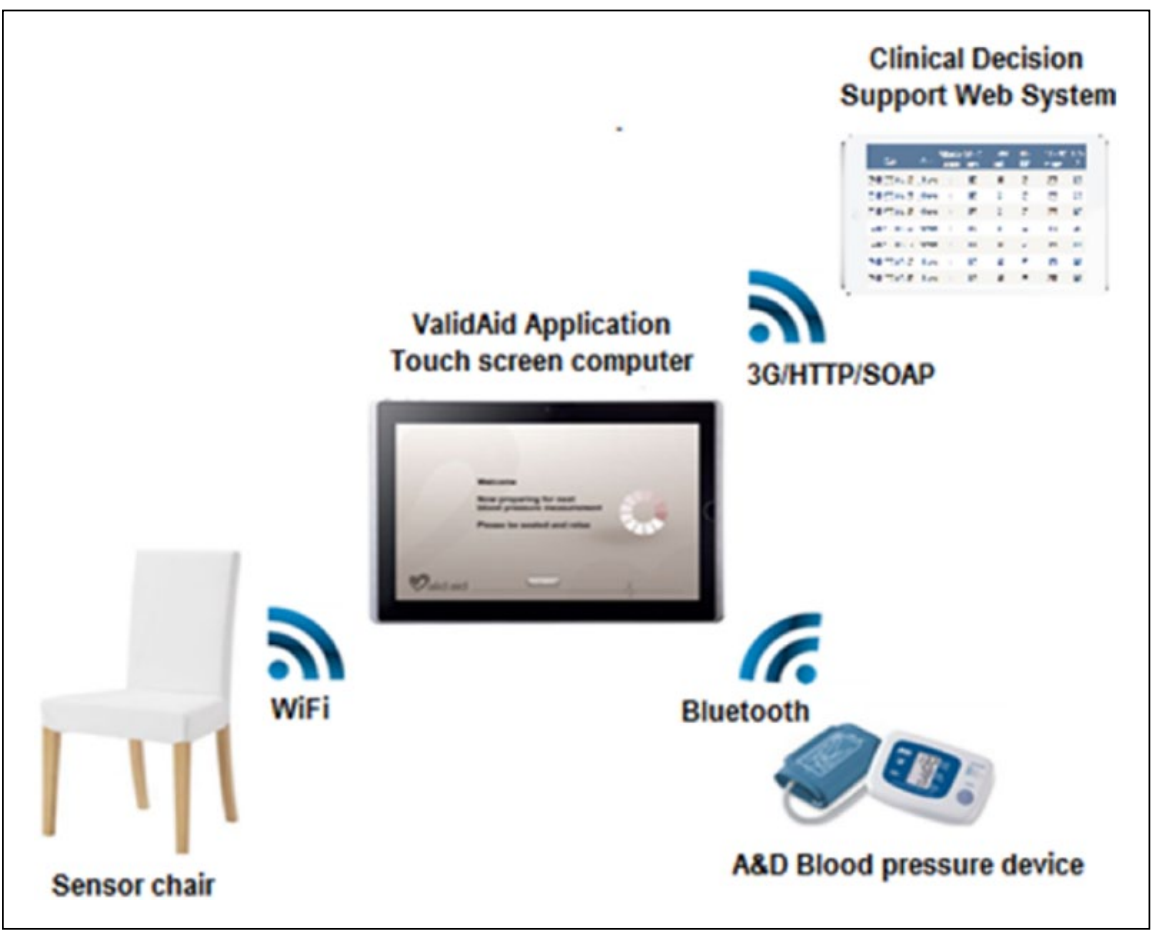

Figure I. Overview of the ValidAid platform, consisting of a context-aware sensor chair based on piezo-resistive sensors, a touch screen computer running the ValidAid application. Finally, the A\&D blood pressure device is connected using Bluetooth. The chair shown is the actual chair used for this study.

sufficient rest time has been achieved. Also, warnings are provided during measurements, for example, if the user speaks during measurements, or if the user moves during the measurement process.

The sensor chair has previously been validated in a laboratory study ${ }^{17}$ and has also been used in a clinical study for measuring patient adherence to BP recommendations without interactive guidance provided, with the participation of 113 kidney disease patients. ${ }^{18}$

The ValidAid audio classification algorithm, which was used to detect talking and ambient noise levels during measurements, is based on machine learning techniques including feature extraction and classification using an artificial neural network classifier to detect patients talking during measurements.

The algorithm has previously been validated and described in a laboratory study ${ }^{15,16,19,20}$ where 80 unique test subjects participated for training purposes and another 20 test subjects were used to validate the performance of the algorithm.

The ValidAid sensor chair has also been validated with regard to its ability to assess whether the patient is correctly seated during measurements, and if they have adhered to the recommended rest time. ${ }^{19}$

The ValidAid system used in the study features both adherence verifiers, as defined in the Adherence Strategy Engineering Framework (ASEF) framework, ${ }^{21}$ for assessing the ability of the patient to follow the required procedure and report non-adherent behavior to the healthcare professional, as well adherence aids ${ }^{21}$ for active and context-aware guidance of the patient which aims to improve adherence levels. 


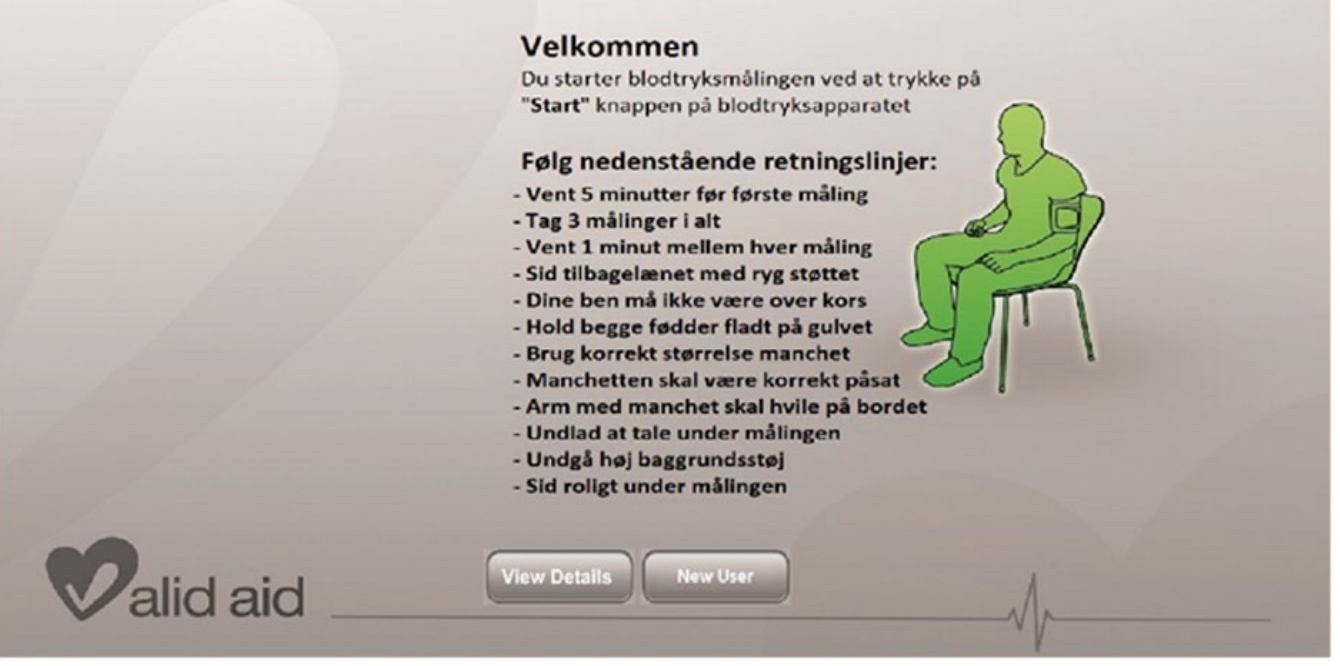

Figure 2. Screenshot from the patient user interface. In the top left corner, we find an active adherence aid that provides a countdown timer indicating when the next measurement should be taken. If a patient performs a measurement prematurely, she is instructed to redo the measurement using on-screen messages. In the center of the screen, the passive adherence aid is located consisting of the list of recommendations that the patient needs to follow in a text-based list format. All screen text is provided in Danish only.

\section{BPSM}

Participants received an anonymous id number and, after careful verbal instruction on how to take reliable BPSM, they were left to autonomously perform three self-measurements in the ValidAid test setup with the aid of a BP device.

They were instructed to be seated comfortably in a quiet area; their arm was supported at heart level, back rested, and legs not crossed. A small $(<22 \mathrm{~cm})$, normal $(22-32 \mathrm{~cm})$, or large $(33-42 \mathrm{~cm})$ adult cuff was used, depending on mid-arm circumference. ${ }^{22}$ Participants were required to rest $5 \mathrm{~min}$ before the first measurement and at least $1 \mathrm{~min}$ between the three measurements.

All participants were actively guided with the aid of a countdown on the tablet computer; they were able to follow how long they had been seated and when to take the three measurements. There were no active context-aware adherence aids used for the remaining context parameters: ambient noise-level, legs-crossed, and talking. These parameters were, however, recorded and stored for future analysis. All measurements were automatically stored and data were made available to staff via a web-based system.

\section{Data analysis}

The primary outcome was the assessment of whether patients would be able to self-measure their BP adhering to internationally established recommendations and to evaluate patient acceptance 
Table I. Background and blood pressure measurement data obtained.

\begin{tabular}{|c|c|}
\hline Variable & Value, mean ( $95 \%$ confidence interval), $\mathrm{p}$-value \\
\hline Age (years) & 29.9 (95\% Cl: 28.9-30.8) \\
\hline Gestational age (weeks) & $12+4(95 \% \mathrm{Cl}: 12.3-12.5)$ \\
\hline Systolic BP, mm Hg & $109.2 \mathrm{~mm} \mathrm{Hg}(95 \% \mathrm{Cl}:$ I07.5-I I0.9) \\
\hline Diastolic BP, mm Hg & $72.3 \mathrm{~mm} \mathrm{Hg}(95 \% \mathrm{Cl}: 70.6-73.9)$ \\
\hline Pulse, beats per minute (BMP) & 79.I bpm (95\% Cl: 77.0-8I.3) \\
\hline Mean arterial pressure (MAP) & $84.6 \mathrm{~mm} \mathrm{Hg}(95 \% \mathrm{Cl}: 83.0-86 . \mathrm{l})$ \\
\hline $\begin{array}{l}\text { Difference between first and second } \\
\text { measurements (MAPI vs MAP2) }\end{array}$ & $0.0 \mathrm{~mm} \mathrm{Hg}(95 \% \mathrm{Cl}: 0.5 ; \mathrm{I} .8), \mathrm{p}<0.05$ \\
\hline $\begin{array}{l}\text { Difference between second and third } \\
\text { measurements (MAP2 vs MAP3) }\end{array}$ & I.I mm Hg (95\% Cl: $-0.4 ; 0.5), p>0.05$ \\
\hline $\begin{array}{l}\text { Difference between third and first } \\
\text { measurements (MAP3 vs MAPI) }\end{array}$ & I. $2 \mathrm{~mm} \mathrm{Hg}(95 \% \mathrm{Cl}: 0.6 ; \mathrm{I} .8), \mathrm{p}<0.05$ \\
\hline
\end{tabular}

levels of performing self-measurements using context-aware adherence aids for guidance. In addition to this, intrapatient differences between the three performed measurements were investigated. For this, we calculated the resulting three mean arterial pressure (MAP) value series based on the recorded BP measurements, performed normality testing using qq-plots and histograms, and then applied three paired t-tests to investigate for differences in means between the three MAP value series. All data analysis was done with MiniTab and verified with Excel. Patients having performed fewer than three measurements were excluded, as well as all measurements recorded after the third measurement of a patient, which were considered as operator errors.

\section{Results}

\section{Participant background and BP measurement data}

We recorded a total of 308 individual measurements from 100 unique patients. Detailed results of patient data, including age, gestational age, systolic and diastolic BP, heart rate, and MAP values can be seen in Table 1. Of these, 300 measurements from 100 patients were used to investigate for intrapatient differences between first, second, and third MAP value series. The mean and confidence interval and corresponding p-value are likewise presented in Table 1.

\section{Measured adherence levels}

In all, 93 percent of participants took the required three measurements. The remaining 7 percent of participants performed either four $(6 \%)$ or five $(1 \%)$ measurements. In six out of the seven cases, each participant took a "premature measurement" prior to the required 5-min rest limit. These measurements were taken within 0-198 s after the patient was first seated. The six participants did, however, eventually manage to take their first measurement after the correct 5-min rest time, achieving three valid BP measurements.

One participant took extra measurements during the rest time between measurements two and three. Participant adherence to the recommendations rest time and not talking was 99 percent. Keeping legs uncrossed was adhered to in 69 percent, while back supported was adhered to by 35 percent of the participants. One percent of participants failed to self-report correct measurements. Table 2 presents the percentage of patients adhering to the individually measured recommendations. 
Table 2. The number of patients adhering to the recommendations during self-measurements while using the ValidAid system to self-measure blood pressure.

\begin{tabular}{ll}
\hline Recommendation adhered to & Patients adhering \\
\hline Performed at least three measurements & $93 \%$ \\
Rest time (5 min) & $99 \%$ \\
Not talking & $99 \%$ \\
Legs not crossed & $69 \%$ \\
Back supported & $35 \%$ \\
Correct self-reporting of BP & $99 \%$ \\
\hline
\end{tabular}

\section{Patient acceptability questionnaire results}

The questionnaire results (shown in Figures 3 and 4) show that 95 percent felt "very comfortable" with self-measurement, 81 percent strongly agreed to this, while 14 percent partly agreed. In all, 17 percent felt "uncomfortable" having to self-measure, while 7 percent expressed strongly agreeing to this. In all, 93 percent disagreed that they felt unnecessarily monitored by the BP equipment, 87 percent strongly disagreeing to this. Eight percent would have preferred that BP measurements were performed by healthcare personnel.

\section{Discussion}

We found that the majority of participants (93\%) took exactly the required three BP measurements. Adherence to the actively enforced recommendations, rest time and not talking, was 99 percent. Adherence to keeping legs uncrossed was 69 percent, while back supported was only adhered to by 35 percent of the participants, which was likely due to these recommendations not being actively enforced by the ValidAid system.

The questionnaire also showed that 95 percent felt comfortable with self-measurement after having used ValidAid, of which 81 percent strongly agreed. This indicates that performing selfmeasurements is perceived as acceptable to the vast majority. In all, 93 percent disagreed that they felt unnecessarily monitored by the BP equipment. Eight percent would have preferred that BP measurements were performed by healthcare personnel. However, this does not imply that they did not feel comfortable with performing self-measurements.

Participants were actively guided, with the aid of a countdown on the monitor. They were able to follow how long they had been seated and know when to take the three measurements. The six participants who performed premature measurements, eventually managed to take their first approved measurement after the correct 5-min rest time, therefore achieving three valid BP measurements. Such extra measurements are of no clinical importance and thus acceptable.

The participants' ability to correct their measurement process after having taken invalid measurements is likely due to the context-aware adherence aid which, upon premature measurement, would inform the participants of the insufficient rest time and instruct them to redo the measurement after the 5-min rest time, but the underlying mechanisms should be studied further.

Participant adherence to recommendations that were enforced with a combination of verbal staff explanations and active interactive guidance aids, rest time and not talking, was observed as being satisfactory achieving 99 percent adherence. However, verbally explained recommendations that were only passively enforced as on-screen guidance showed much lower adherence levels. 


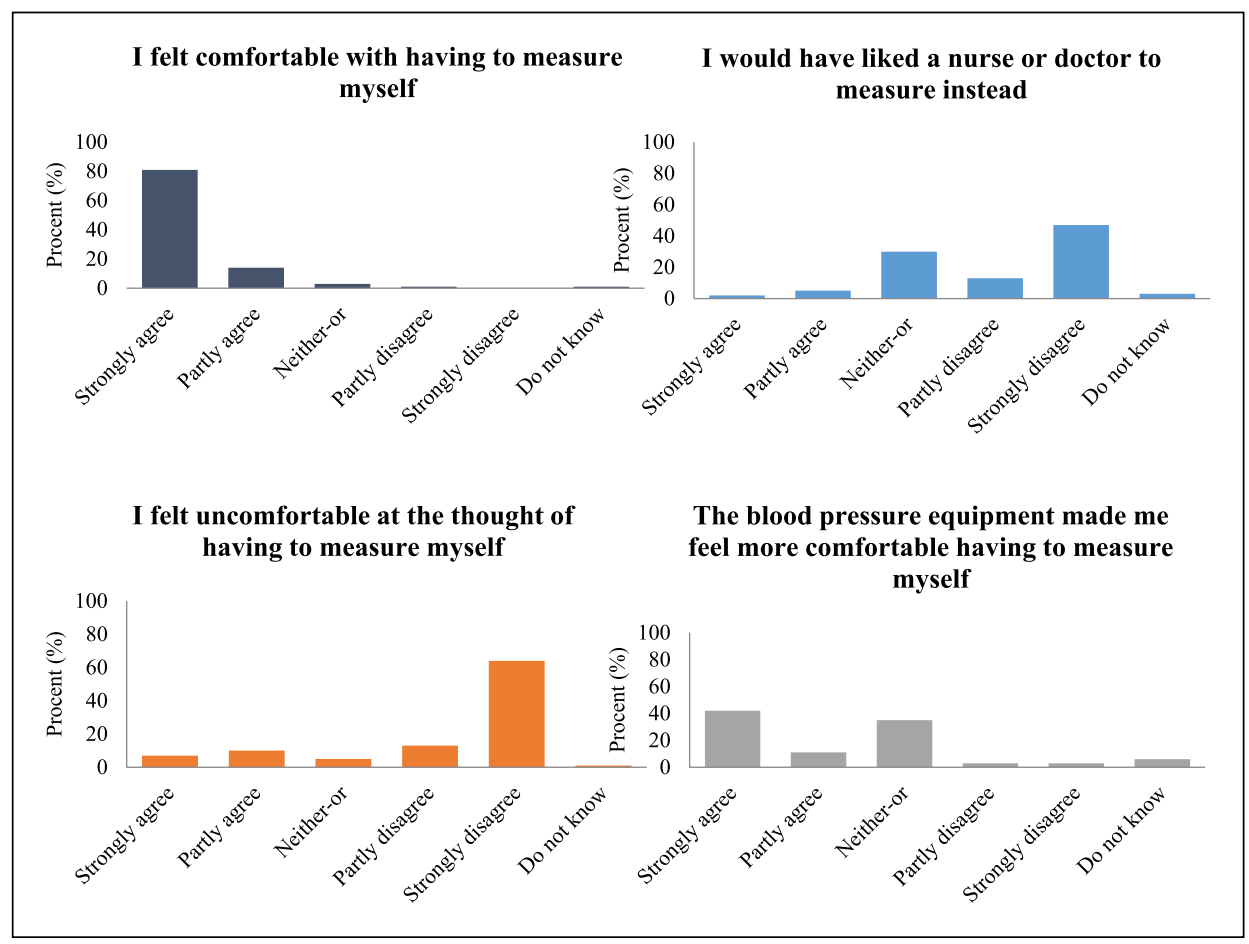

Figure 3. Questionnaire results detailing the participants' experiences with the self-measurement process. The first four questions focused on the patient's initial attitude toward self-measurement, and whether they would have preferred a nurse or doctor instead of a sensor-based self-measurement station, and finally whether they felt comfortable having to measure themselves using the ValidAid context-aware self-measurement station.

This indicates that active guidance aids are more effective in achieving adherence than passive aids. The participant's inadequacy to adhere to the recommendations may cause critical bias. ${ }^{13}$ Further studies are needed to detail the underlying mechanisms.

Three paired t-tests were performed on the recorded MAP values, in order to investigate any differences in means between the first, second, and third measurement sets. Although the t-tests did indicate a difference in means between the first and the second MAP series and the first and the third MAP series ( $\mathrm{p}<0.05$ for both), the actual differences were well below the typical variability of BP measurements that is commonly accepted (up to $5 \mathrm{~mm} \mathrm{Hg}$ is not considered a difference due to BP variability and device precision). For both the relevant MAP comparisons, the differences in mean were below $1.3 \mathrm{~mm} \mathrm{Hg}$ and with a 95 percent confidence interval between 0.5 and $1.8 \mathrm{~mm} \mathrm{Hg}$ (see Table 1). Thus, no practical difference could be found between the three paired MAP values, and all three measurements series are thus assumed to be equal in means.

The ValidAid system was designed to provide active guidance in the shape of a context-aware adherence aid ${ }^{21}$ with regard to rest time only; all other parameters were not provided for. Whether context-aware adherence aids could be beneficial, if introduced to all of the recommendations, would need to be verified further, but appears likely. Specifically, we passively collected data on the participants ability to keep legs uncrossed and back supported, but we did provide an interactive context aid, reminding the participants to "sit back" and "uncross legs" once such erroneous 


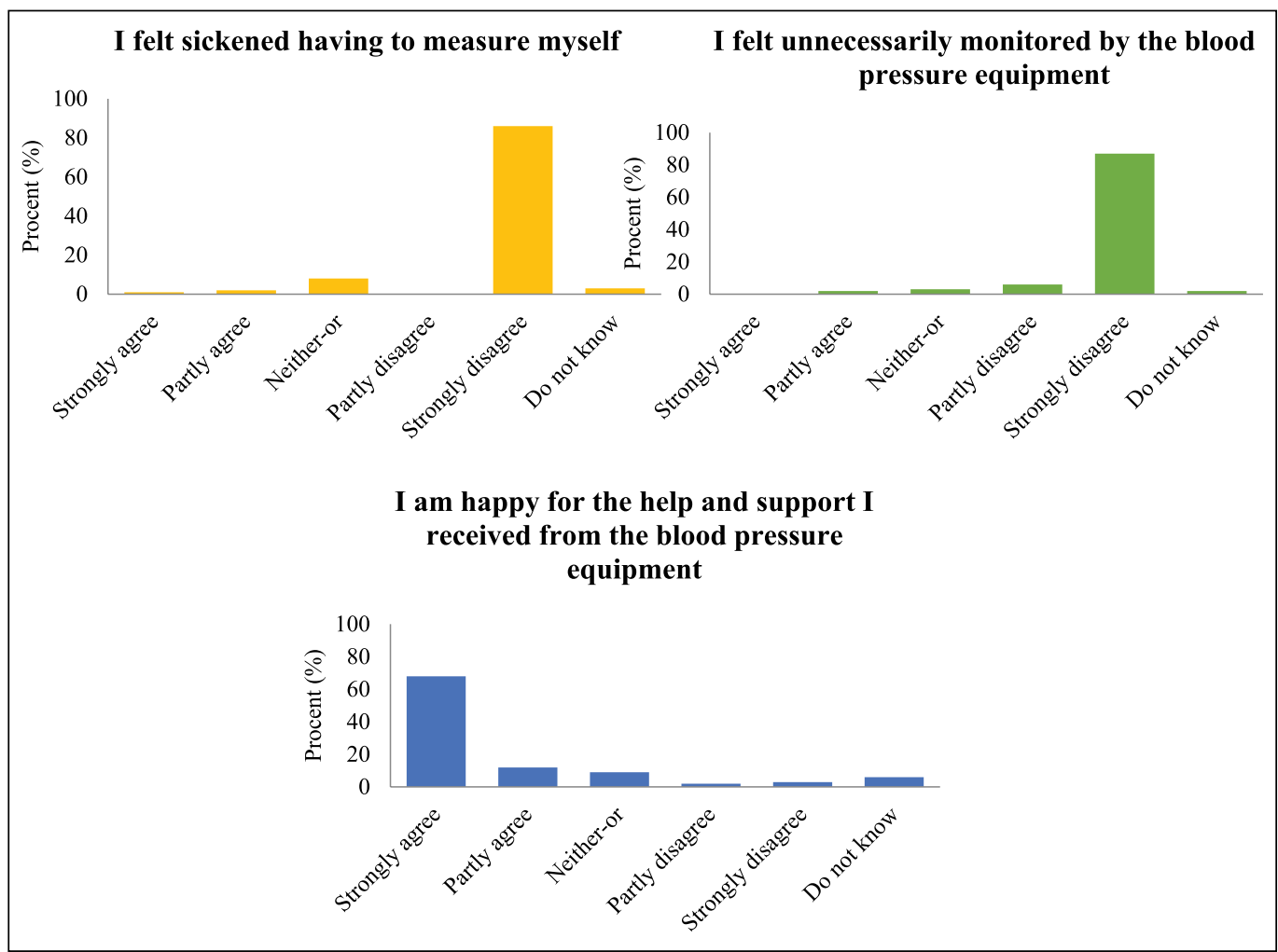

Figure 4. Questionnaire results detailing the participants' experiences with the self-measurement process. The last three questions focused on the patient's experience after having completed the selfmeasurement process. This includes whether the self-measurement process made them feel needlessly sickened (stigmatized) as patients, and whether they felt overly monitored by the extra sensors. The final question focused on whether the participants felt they had received sufficient support from the system.

behavior was detected. This was, in part, due to not wanting to overwhelm the participants with interactive advice during this initial study. However, these two interactive aids should be implemented in future self-measurement systems, if possible.

It could be argued, that a basic countdown timer could be sufficient for achieving relevant rest time of the participants. However, in a previous study, where only a simple clock was provided for countdown and no active adherence aid was used, we found that only 8 percent of the patients adhered to the required rest time of $5 \mathrm{~min}$ before taking the first measurement; less than half of all measurements, including the second and third measurement, were performed after the required 5-min rest time. ${ }^{18}$ Existing BP devices and tele-monitoring systems are not equipped to detect non-adherent behavior. Thus, in order to validate measurements taken with state-of-theart equipment, it is necessary for manual staff observation in order to guarantee the quality of the measurements. Relying on manual observation would require increased staff resources and would therefore not be cost-effective.

Patients could, alternatively, be equipped with telemedicine devices, featuring relevant contextaware aids to self-measure in the comfort of their homes. This approach would, however, incur increased costs in regard to both equipment and maintenance. 
Our results showed that only one percent failed to self-report correct measurements, which is very low compared to related studies. Previous studies have found that up to 50 percent of all the self-reported BP data did not match the data stored in device memory. ${ }^{18,23-25}$ This difference could be explained by the study setup, where measurements were indicated both on the touch screen and on the BP device itself. In the related studies, the participants were not aware that the measurement was being automatically recorded.

Furthermore, it could be speculated that an active guidance system, such as ValidAid, could have a negative effect on some participants in terms of building up anxiety levels. Being continuously corrected by the ValidAid system (or similar active guidance systems) might result in increased anxiety, which is well known to be associated with artificially increased BP values. ${ }^{19}$ In other words, it could be speculated whether the increased use of technology could result in anxiety effects similar to the "white coat effect."19

These results, combined with the findings on participant adherence levels to the recommendations, indicate the relevance of introducing a self-measurement tool with active adherence guidance aids to support the participants.

Our results are in line with previous work, ${ }^{18,23-26}$ which likewise found that adherence improves when using active and context-aware adherence aids. ${ }^{21}$ However, the work presented in this article is the first to study the effects of patient adherence to recommendations when using active adherence aids in a large cohort of patients, thus demonstrating a novel application of context-aware sensors in a smart environment clinical healthcare scenario. Specifically, clinical procedures with regard to the screening of PE currently relies on manual BP measurements which is resource consuming and impractical for large-scale screening efforts. ${ }^{6}, 11$ With selfmeasurement stations, such as ValidAid, our results indicate that automated self-measurement is feasible and safe, although more work is needed in order to refine this procedure. As a further novelty, we have investigated the user experience of patients being exposed to a self-measurement system, which has not been reported elsewhere in the literature to our knowledge. The user experience is of pivotal importance, as a negative user experience could cause bias to readings and hamper proliferation efforts further. Thus, more work is needed in order to determine the optimal level of active adherence aids and verifiers used to achieve accurate measurements and self-measurement user experience. This work should be undertaken in the shape of randomized controlled experiments in one or more clinical settings with a sufficient patient population in order to provide sufficient statistical power.

Finally, the use of self-measurement stations relying on a combination of context and medical sensors could be extended to other areas, including in the telemedicine and ambient-assisted living settings. ${ }^{27,28}$

\section{Conclusion}

It was found that the majority of the participating healthy pregnant women were able to take reliable BPSMs using the BP self-measurement station ValidAid, adhering to internationally established BP measurement recommendations. Thus, we found it feasible to use the context-aware system ValidAid, to improve self-measurement adherence.

There was an interesting difference in the degree of compliance between the recommendations that were actively enforced $(93 \%-99 \%)$ contra those that were not enforced $(35 \%-65 \%)$. Participants generally complied when being actively guided by adherence aids (including time seated at $99 \%$ ), while compliance to those not enforced was low (back supported at $35 \%$ and legs not crossed at $69 \%$ ). 
The majority of the participants felt comfortable having to self-measure, while only a small percentage would have preferred the measurements to be taken by healthcare staff. Further development of the interactive features of the platform may potentially facilitate increased adherence to the recommendations and reduce insecurity related to self-measurement.

\section{Acknowledgements}

Camilla Lindahl, Stefan Wagner, Niels Uldbjerg, Jacob M. Schlütter and Puk Sandager jointly conceived and designed the experiments; Stefan Wagner built and validated the ValidAid research prototype; Camilla Lindahl, Stefan Wagner, Niels Uldbjerg, Jacob M. Schlütter, Puk Sandager, and Olav W. Bertelsen jointly conceived the qualitative questionnaire; Camilla Lindahl, Stefan Wagner, Niels Uldbjerg, Jacob M. Schlütter and Puk Sandager analyzed the data; Camilla Lindahl performed the experiments; and Camilla Lindahl, Stefan Wagner, Niels Uldbjerg, Jacob M. Schlütter, Puk Sandager, and Olav W. Bertelsen jointly wrote the paper, with Camilla Lindahl, Puk Sandager, and Stefan Wagner acting as main contributors and Camilla Lindahl as first author.

\section{Declaration of conflicting interests}

The author(s) declared no potential conflicts of interest with respect to the research, authorship, and/or publication of this article.

\section{Funding}

The author(s) disclosed receipt of the following financial support for the research, authorship, and/or publication of this article: This work was supported by a Danish national government grant from the Ministry of Higher Education and Science Grant Number: 1234-12345B_VK.

\section{References}

1. Duley L. The global impact of pre-eclampsia and eclampsia. Semin Perinatol 2009; 33(3): 130-137.

2. Geographic variation in the incidence of hypertension in pregnancy. World Health Organization international collaborative study of hypertensive disorders of pregnancy. Am J Obstet Gynecol 1988; 158(1): $80-83$.

3. Khan KS, Wojdyla D, Say L, et al. WHO analysis of causes of maternal death: a systematic review. Lancet 2006; 367(9516): 1066-1074.

4. Killion M. New hypertension in pregnancy guidelines. MCN Am J Matern Child Nurs 2015; 40(2): 128.

5. Duley L. Pre-eclampsia, eclampsia, and hypertension. BMJ Clin Evid 2011; 2011: 1402.

6. Akolekar R, Syngelaki A, Poon L, et al. Competing risks model in early screening for preeclampsia by biophysical and biochemical markers. Fetal Diagn Ther 2013; 33(1): 8-15.

7. Nicolaides KH. Turning the pyramid of prenatal care. Fetal Diagn Ther 2011; 29(3): 183-196.

8. Bujold E, Roberge S, Lacasse Y, et al. Prevention of preeclampsia and intrauterine growth restriction with aspirin started in early pregnancy: a meta-analysis. Obstet Gynecol 2010; 116(2 Pt 1): 402-414.

9. Roberge S, Villa P, Nicolaides K, et al. Early administration of low-dose aspirin for the prevention of preterm and term preeclampsia: a systematic review and meta-analysis. Fetal Diagn Ther 2012; 31(3): 141-146.

10. Park F, Russo K, Williams P, et al. Prediction and prevention of early onset pre-eclampsia: the impact of aspirin after first trimester screening. Ultrasound Obstet Gynecol 2015; 46: 419-423.

11. Poon LC and Nicolaides KH. Early prediction of preeclampsia. Obstet Gynecol Int 2014; 2014: 297397.

12. O’Brian E, Asmar R, Beilin L, et al. European Society of Hypertension recommendations for conventional, ambulatory and home blood pressure measurement. J Hypertens 2003; 21(5): 821-848.

13. Pickering TG, White WB, Giles TD, et al. When and how to use self (home) and ambulatory blood pressure monitoring. J Am Soc Hypertens 2010; 4(2): 56-61. 
14. Helvaci MR and Seyhanli M. What a high prevalence of white coat hypertension in society. Intern Med 2006; 45(10): 671.

15. Wagner S, Kamper C, Rasmussen N, et al. Reliable blood pressure self-measurement in the obstetric waiting room. Methods Inf Med 2014; 53(3): 225-234.

16. Mancia G, Fagard R, Narkiewicz K, et al. 2013 ESH/ESC Guidelines for the management of arterial hypertension. J Hypertens 2013; 31(7): 1281.

17. Wagner S, Toftegaard TS and Bertelsen OW. Challenges in blood pressure self-measurement. Int $J$ Telemed Appl 2012; 2012: 437350.

18. Wagner S, Ahrendt P, Toftegaard TS, et al. Audio context classification for determining blood pressure self-measurement adherence. In: Proceedings of the IADIS International Conference on e-Health, Lisbon, Portugal 17-19 July 2012.

19. Wagner S, Toftegaar, TS and Bertelsen OW. Context assessment during blood pressure self-measurement utilizing the sensor chair. In: International Joint Conference on Ambient Intelligence. Berlin Heidelberg: Springer, 2011.

20. Wagner S Toftegaard TS and Bertelsen OW. Introducing the Adherence Strategy Engineering Framework (ASEF). Methods Inf Med 2013; 52(3): 220-230.

21. Wagner S, Bertelsen OW and Toftegaard TS. The Adherence Strategy Engineering Framework (ASEF).

22. Pickering TG, Hall JE, Appel LJ, et al. Recommendations for blood pressure measurement in humans and experimental animals. Part 1: blood pressure measurement in humans, a statement for professionals from the Subcommittee of Professional and Public Education of the American Heart Association Council on High Blood Pressure Research. Circulation 2005; 111(5): 697-716.

23. Johnson KA, Partsch DJ, Rippole LL, et al. Reliability of self-reported blood pressure measurements. Arch Intern Med 1999; 159(22): 2689-2693.

24. Mengden T, Hernandez Medina RM, Beltran B, et al. Reliability of reporting self-measured blood pressure values by hypertensive patients. Am J Hypertens 1998; 11(12): 1413-1417.

25. Santamore WP, Homko CJ, Kashem A, et al. Accuracy of blood pressure measurements transmitted through a telemedicine system in underserved populations. Telemed J E Health 2008; 14(4): 333-338.

26. Sandager P, Lindahl C, Schlütter JM, et al. Context-aware patient guidance during blood pressure self-measurement. In: Proceedings of the IADIS International Conference E-health 2013, Eh 2013. University of Twente, 2013.

27. Larsen SB, Sørensen NS, Petersen MG, et al. Towards a shared service centre for telemedicine: telemedicine in Denmark, and a possible way forward. Health Informatics $J$ 2016; 22(4): 815-827.

28. Linskell $\mathrm{J}$ and Bouamrane MM. Assisted-living spaces for end-users with complex needs: a proposed implementation and delivery model. Health Informatics $J$ 2012; 18(3): 159-170. 